\title{
Capturing and evaluating blinks from video-based eyetrackers
}

\author{
Xianta Jiang • Geoffrey Tien • Da Huang • \\ Bin Zheng • M. Stella Atkins
}

Published online: 28 December 2012

(C) Psychonomic Society, Inc. 2012

\begin{abstract}
Blinks are related to several emotional states, and the present report describes a simple, reliable way to measure blinks from the video stream of an eye obtained during eyetracking, where the source of the eye video is a video camera attached to a head-mounted eyetracker. Computer vision techniques are employed to determine the moments that a blink starts and ends, for the purpose of calculating blink frequency and duration. The video is first processed to show blocks of eyelid and pupil movements, and is then analyzed for blink starts and ends. The moment of a blink start is reported when the eyelid starts to move quickly, exceeding a predetermined threshold. The end of a blink arises when the pupil size increases by less than a separate threshold. We observed several different blink patterns from different subjects, and our algorithm was designed to work for all of these patterns. We evaluated our algorithm by manually measuring the true blinks of five different subjects while they were eyetracked. To test the sensitivity and specificity of the algorithm, we employed a series of threshold values to plot the receiver operating characteristic curves. Using the best thresholds, we achieved excellent sensitivity ( $>90 \%$ ) and specificity ( $>99 \%$ ) over the five subjects. Potential applications of this research include real-time, nonintrusive, continuous and automated measurements of mental workload and other emotional states related to blink rates and durations.
\end{abstract}

\section{Jiang}

College of Computer Science and Technology, Zhejiang

University, Hangzhou, China

X. Jiang $\cdot$ G. Tien $\cdot$ D. Huang $\cdot$ M. S. Atkins $(\bowtie)$

School of Computing Science, Simon Fraser University, Burnaby, BC V5A 1S6, Canada

e-mail: stella@sfu.ca

B. Zheng

Department of Surgery, University of Alberta, Edmonton, AB, Canada
Keywords Blinks · Eyetracking $\cdot$ Computer vision · Video processing $\cdot$ Image-guided tasks $\cdot$ Mental workload

Blink is an important eye motion, and involuntary eye blinks are controlled by a central mechanism that is often associated with the status of mental states such as fatigue, lapses of attention, and stress (Andreassi, 2000; Marshall, 2007; Ryu \& Myung, 2005). Because of this, blink rates and durations have been used in various studies - for instance, to check the drowsiness of individuals in everyday life (Caffier, Erdmann \& Ullsperger, 2003) or evaluate the mental workloads of drivers (Benedetto, Pedrotti, Minin, Baccino, Re \& Montanari, 2011; Haak, Bos, Panic \& Rothkrantz, 2009; Smith, Shah \& Lobo, 2000) and pilots (Veltman \& Gaillard, 1996, 1998).

Eye blinks are also useful in diagnosing the mental status of patients suffering from neurological disorders (Deuschl \& Goddemeier, 1998; Grauman, Betke, Gips \& Bradski, 2001), in that a reduced rate of eye blinking is associated with Parkinson's disease, and excessive blinking may indicate the onset of Tourette's syndrome, strokes, or other disorders of the nervous system (Deuschl \& Goddemeier, 1998). Furthermore, real-time eye-blink detectors are used as an alternative channel for severely disabled people to interact with computers (Grauman et al., 2001).

To capture blinks, researchers have used methods including electrooculography (EOG; Kong \& Wilson, 1998; Ryu \& Myung, 2005; Veltman \& Gaillard, 1998), electromyography (EMG; Blumenthal, Cuthbert, Filion, Hackley, Lipp \& Van Boxtel, 2005), electroencephalography (EEG; Haak et al., 2009), and other, custom-designed instruments, such as an infrared light beam (Caffier et al., 2003), magnetic coils (Evinger, Manning \& Sibony, 1991), and an optoelectronic motion detector (Mitalis \& Druss, 1985). Pedrotti, Lei, Dzaack and Rötting (2011) developed a data-driven eye-blink detection algorithm for $50-\mathrm{Hz}$ eyetracking protocols, where the pupil diameter was used for correcting 
artifacts before estimating blink start and end. Our research group is also developing a technique to capture blinks from the output of an eyetracker, on the basis of missing data frames saved in eyetracker text output recorded by a Tobii 1750 remote eyetracker. However, these methods of calculating blinks have their shortcomings, as the missing data used for computing blinks are blended with other events that cause data loss during eyetracking, such as large head movements, lack of data quality, and tracking recovery delays. Therefore, any algorithm built on counting missing data may exclude true blinks, on the basis of too long a duration, and may also include false blinks, which are nonblink events that are mistaken for blinks.

Blinks can be manually counted accurately from a webcam video capturing eye movements. However, this is a time-consuming task. We have developed a computer vision algorithm to count blinks automatically from such videos.

Several studies have reported eye-blink detection methods using video analysis techniques (Grauman et al., 2001; Lalonde, Byrns, Gagnon, Teasdale \& Laurendeau, 2007; Moriyama, Kanade, Cohn, Xiao, Ambadar, Gao \& Imanur, 2002; Smith et al., 2000; Wu \& Trivedi, 2008). For example, Lalonde et al. recorded drivers' eye motions in a driving simulator with a specialized near-infrared video camera. The occurrence of blinks was detected on the basis of scaleinvariant feature transform (SIFT) features from very-lowcontrast images. Lalonde et al. reported a $97 \%$ detection rate on blinks; however, they were unable to report the durations of the blinks.

Sukno, Pavani, Butakoff and Frangi (2009) created an algorithm capable of reporting blink frequency and duration from webcam videos. First, they used a face detection technique to find the face area for drivers recorded in a video image; the face area was then segmented to produce the outlines of eyes and other landmarks in the face. Eye blinks were detected when the distance between the eye and other face landmarks changed above a threshold value. Besides blink rate, this algorithm tracked the durations of eyes being open and of full closures of the eyes. Sukno et al. reported a $97.1 \%$ accuracy rate when compared to manual examination of the video database. Since this method was based on face detection, accuracy would drop when the face was undetectable due to large head movements.
Smith et al. (2000) developed methods to detect eye occlusions caused by large head movements while driving in a car simulator and to separate these occlusions from naturally occurring blinks. Smith et al. detected the color difference between skin on the face and the sclera in the eye. When the participant's head turned, the sclera in the eye would no longer be detected. This could be used for adjusting reports on blink frequency.

However, face detection techniques have limitations. The eye area is relatively small in the image captured by a webcam, so it is hard to calculate the blink duration accurately (Lalonde et al., 2007; Moriyama et al., 2002; Smith et al., 2000).

We describe here a new algorithm designed to detect blinks using a computer vision algorithm. Recently, in a study on surgeons' vigilance in the operating room (Tien, Atkins, Zheng \& Swindells, 2010; Zheng, Jiang, Tien, Meneghetti, Panton \& Atkins, 2012; Zheng, Tien, Atkins, Swindells, Tanin, Meneghetti \& Panton, 2011), we recorded the eye motions of surgeons wearing head-mounted eyetrackers (PT Mini, Locarna System Inc, Victoria, BC). The eyetracker consisted of an infrared camera pointing to the wearer's eye, designed to capture the motion of the eyeball, and another camera pointing forward, which captured the scene observed by the wearer (Fig. 1a). We describe here a simple, reliable way to measure blinks from the video graphic array (VGA) video stream of the eye obtained during eyetracking, where the source of the eye video is a video camera attached to a head-mounted eyetracker. Through postprocessing, we applied computer vision techniques to these videos to open a new opportunity for us to retroactively identify the blinks from the eyetracking video.

There are several advantages of employing computer vision techniques for capturing eye blinks from the eye camera of a head-mounted eyetracker. First, the camera is set to focus on the center of the eye, and the image is recorded using infrared light, which provides constant illumination under varying conditions. The quality of the image is high, which makes it easier to capture blinks. Second, the contrast of the image is sufficient to separate different components of the eye. As is shown in Fig. 1b, the pupil is the darkest part in the image and has a relatively brighter ring around it (the iris), so we can easily separate the pupil from the remaining parts of the eye. Third, the eyelid moves speedily during the start of a blink (Caffier et al., 2003).
Fig. 1 (a) The Locarna PTMini eyetracker and (b) a screenshot of the video graphic array image of the subject's eye

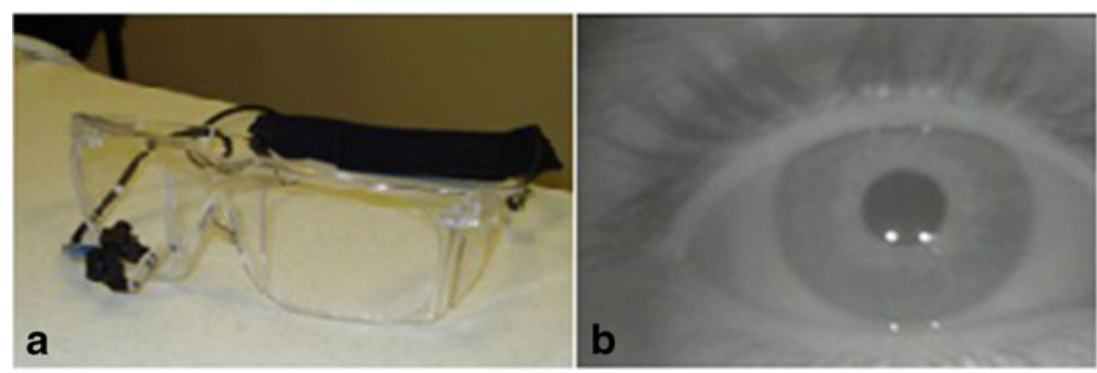


To identify the best parameter settings and test the sensitivity and specificity of the algorithm, a series of threshold values were used, and receiver operating characteristic (ROC) curves were plotted.

\section{Method}

\section{Experimental setting}

Surgeons were asked to perform a simulated surgical task in a virtual reality simulator (METI SurgicalSim). Their eye motions were recorded using a wearable head-mounted eyetracker (Locarna PT Mini). The task and apparatus were designed for studying surgeons' vigilance in the operating room. The details of the experimental setting are given elsewhere (Tien et al., 2010), and the results are in Zheng et al. (2012).

\section{Observation of the videos}

While reviewing the videos, we observed that individuals have different blink patterns. We saw that most individuals' blinks fell into one of two categories; completely closed eye blinks, in which the upper eyelid would drop completely over the pupil during a blink, and partially closed eye blinks, in which the upper eyelid would barely drop over the top of the pupil. Some partially closed eye blinkers even have blinks in which the upper eyelid fails to touch the pupil during blinking. Five different blink patterns are shown in Fig. 2, in which each column corresponds to successive frames of a subject during a blink. The first two columns show subjects with completely closed eye blinks, and the other three show subjects with partially closed eye blinks. Analysis of these videos and natural observations convinced us that these partially closed eye blinks did not arise as an artifact of the relatively low $(30-\mathrm{Hz})$ rate of sampling.

On the basis of these facts, we developed a robust algorithm for computing the start and end of eye blinks from the output videos of head-mounted eyetrackers. Our algorithm was designed to work on all of these patterns. Once blinks were identified, we reported blink frequency and duration for the analysis of mental workload.

\section{Video processing}

We developed a computer algorithm to process videos to capture blinks using $\mathrm{C}++$ (Microsoft Visual Studio) and Open Computer Vision Library (Open CV; Bradski \& Kaehler, 2008). As is shown in Fig. 1a, two mini cameras are mounted on the Locarna eyetracker: One records the motion of the right eye of the wearer; this is an infrared camera, which is the video source used in the present work. The other camera records the scene that the wearer is looking at.
The video was originally saved in grayscale color mode with a resolution of $352 \times 240$ pixels at $30 \mathrm{~Hz}$. The video was read in and processed frame by frame using Open CV functions.

Figure 3, column 1, shows consecutive frames from the eyetracker video during a typical completely closed eye blink (the same subject shown in column 2 in Fig. 2).

Algorithm for detecting the start of a blink

The start of a blink is detected by examining the difference between the current and the previous frame. The first step is to calculate the grayscale difference image between the current and the previous frames:

$I_{\text {diff }}=I_{\text {cur }}-I_{\text {pre }}$.

In Eq. $1, I_{\text {cur }}$ and $I_{\text {pre }}$ are the grayscale images of the current and the previous frame, and $I_{\text {diff }}$ is the difference grayscale image between $I_{\text {cur }}$ and $I_{\text {pre }}$.

The second step is to convert $I_{\text {diff }}$ to a binary image using a threshold $\theta_{\mathrm{d}}$ :

$I_{\text {bin }}(x, y)=\left\{\begin{array}{ll}I_{\text {diff }}(x, y)<\theta_{d} & 0 \\ I_{\text {diff }}(x, y) \geq \theta_{d} & 255\end{array}\right.$.

$\theta_{\mathrm{d}}$ was determined using the method described in the Evaluation of Algorithm section below. To make the binary image $I_{\text {bin }}$ more structured, morphological image processing (erosion and dilation) was applied (Bradski \& Kaehler, 2008), with a grid-shaped structuring element (three columns and three rows) in opening operation mode (by first doing erosion and then dilation). The erosion operation removes pixels on the boundaries of objects in the input image according to the structuring element, while dilation adds pixels on the object boundaries. These two operations make the edge of the object in the image more smooth and structured.

In these binary images, two important eye structures were recognized: the eyelid and pupil. The blink start was identified by examining the position changes of the eyelid on two consecutive image frames. As is shown in the second column in Fig. 3, when the blink started, the difference in eyelid position between the third and the second frames is large, so it can be used for identifying the start of the blink.

The third step is to find the biggest connected object in $I_{\text {bin. }}$. If the ratio of the width of the found object and the width of the eye area is larger than a threshold $\theta_{\mathrm{s}}$, the blink is considered to have started. An illustration of the step for detecting the blink start is shown in Fig. 4.

Algorithm for detecting the end of a blink

The end of the blink is detected by the change of pupil size after the start of a blink. The pupil is the darkest part recorded 


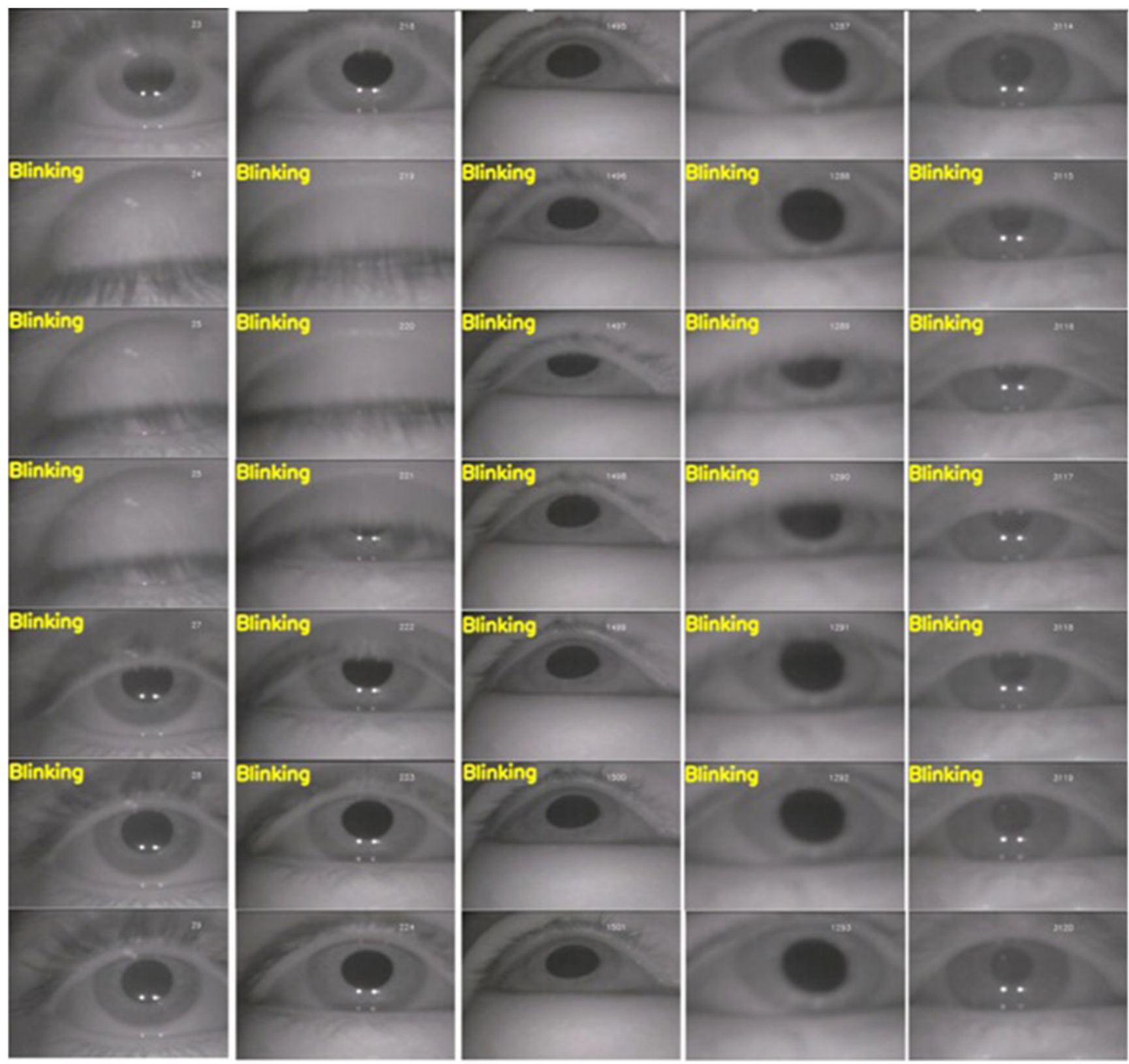

Fig. 2 Successive screenshots from videos of five different blink patterns. The first two columns have completely closed eye blinks, and the other three columns have partially closed eye blinks

in the video image. To detect the pupil, the grayscale image is converted into a binary by applying a preset threshold $\theta_{\mathrm{p}}$ according to Eq. 3 . The pupil then is separated from the remaining parts of the eye. The method of determining $\theta_{\mathfrak{p}}$ will be described in the next section. After getting the binary image $I_{\text {bin }}$, morphological image processing (erosion and dilation) similar to that for detecting the blink start is applied. Finally, we find the biggest connected object in the image.

$I_{b i n}(x, y)= \begin{cases}I_{c u r}(x, y)<\theta_{p} & 255 \\ I_{c u r}(x, y) \geq \theta_{p} & 0\end{cases}$
In order to judge whether a detected connected object is the pupil, we set up criteria to define pupil size, position, and shape. Within these criteria, the width of the detected object must be larger than a certain ratio to the entire image; the location of the object cannot be too close to the edge of the image; and the width-toheight ratio of the object needs to be maintained between 0.3 and 3 .

As is shown in the third column of Fig. 3, the height of the pupil decreases as the eyelid drops from the second to the third row. The pupil disappears for several frames (rows 4-6) when the eyes are completely closed. The pupil then 
Fig. 3 A series of consecutive frames from the Locarna eyetracker video during a typical completely closed eye blink. The left column shows the original VGA image. The blink is detected and labeled by yellow text. The central column shows the binary image of the upper eyelid movement, and the right column shows a binary image in which the pupil is extracted

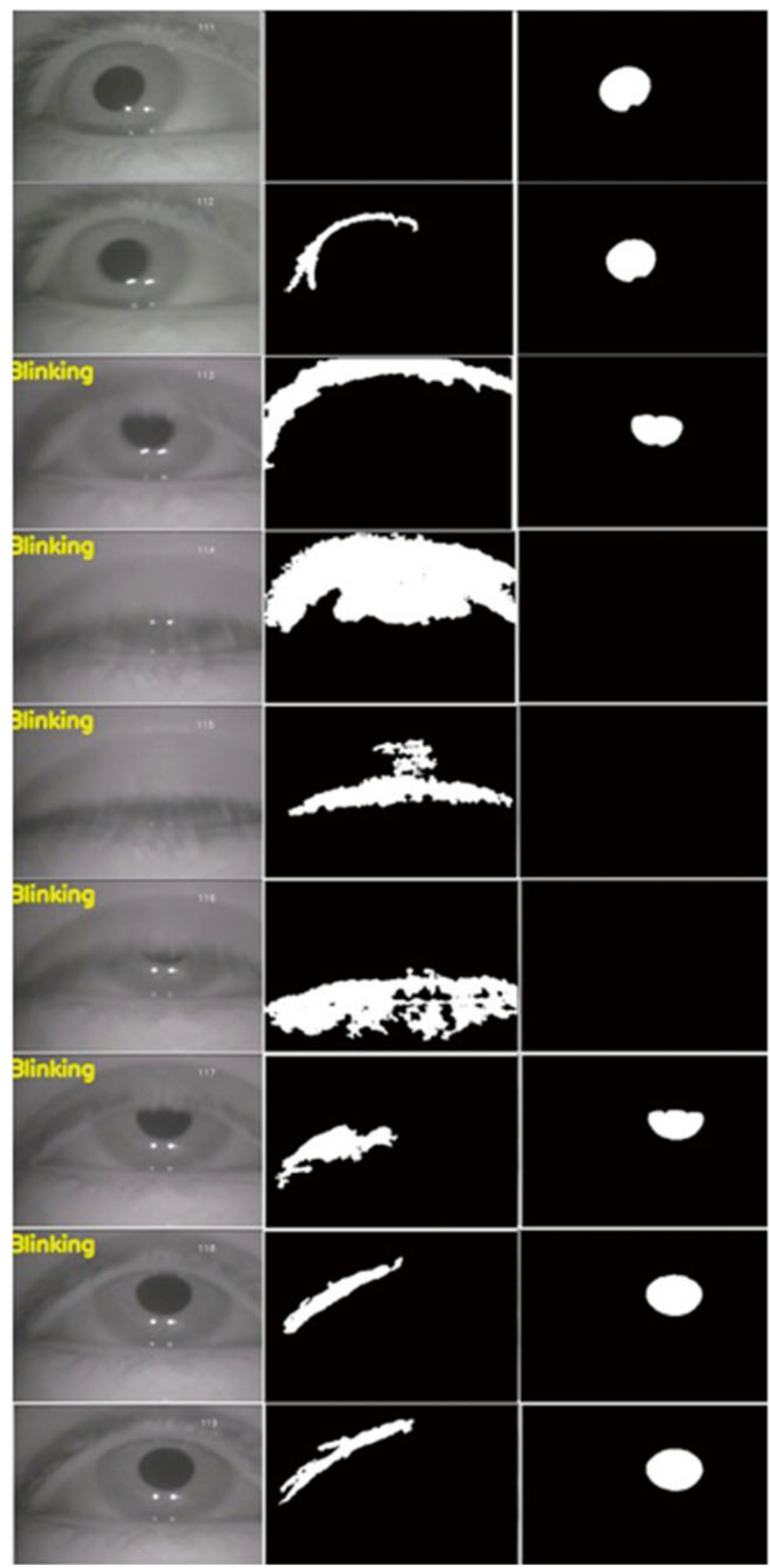




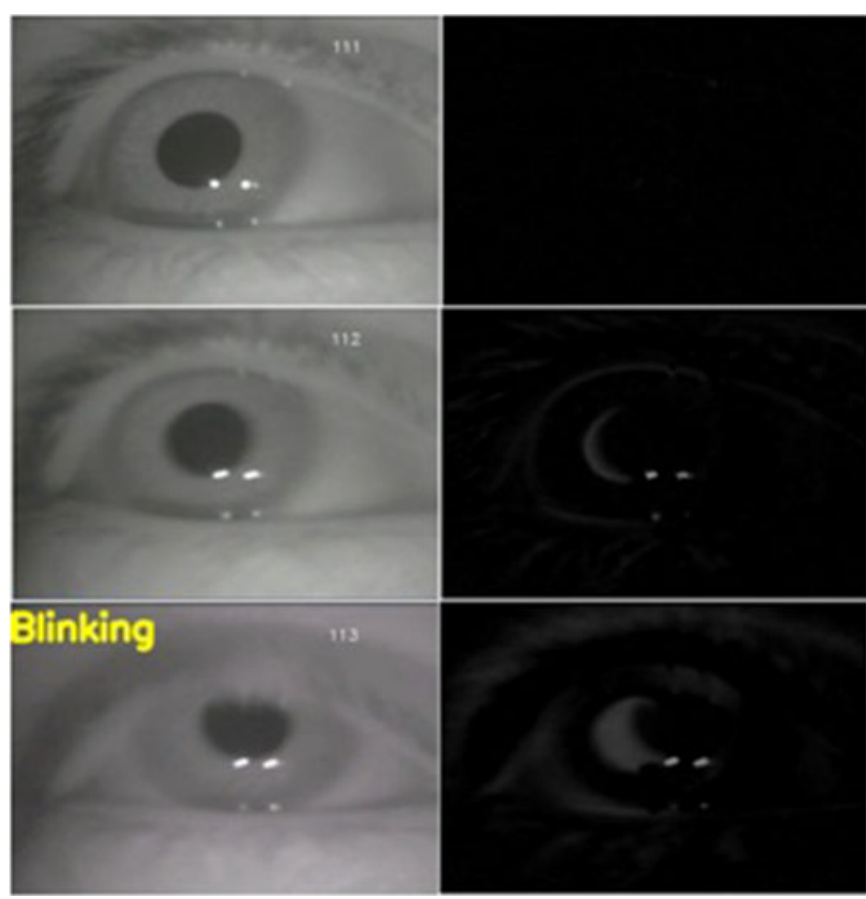

Fig. 4 Steps for detecting the start of a blink. The first column contains the original video graphic array image; the image that results from subtracting each two consecutive frames is shown in the second

reappears and the size increases (rows 7-9) during the reopening phase. Therefore, following an eyelid drop, when the pupil height does not increase above a threshold $\theta_{\mathrm{e}}$ (pixels), the end of the blink is recorded.

For partially closed eye blinks, if the upper eyelid fails to touch the pupil, this means that the change of pupil size is not suitable for determining the end of an eye blink. In these situations, we ended the blink in three frames $(90 \mathrm{~ms})$, because these blinks usually are relatively short; normally, the blink duration is longer than $100 \mathrm{~ms}$ (Schiffman, 2000).

\section{Evaluation of algorithm}

It is clear that the values of $\theta_{\mathrm{s}}, \theta_{\mathrm{e}}, \theta_{\mathrm{d}}$, and $\theta_{\mathrm{p}}$ will affect the detection of blinks. A purpose of this study was to find the best value of $\theta_{\mathrm{s}}, \theta_{\mathrm{e}}, \theta_{\mathrm{d}}$, and $\theta_{\mathrm{p}}$, so that computer-reported blinks would match true blinks.

To verify the accuracy of the algorithm and to choose the best $\theta_{\mathrm{s}}, \theta_{\mathrm{e}}, \theta_{\mathrm{d}}$, and $\theta_{\mathrm{p}}$, ten videos from five different subjects (each of whom performed two trials) with different blink patterns, from the experiment described in the Experimental Setting section, were watched by an experimenter to get the true blink values, in terms of frequency and duration. Typical screenshots of the five subjects' videos are shown in Fig. 2.

We observed the videos frame by frame for true blinks. The start and end criteria of blinks are as below:

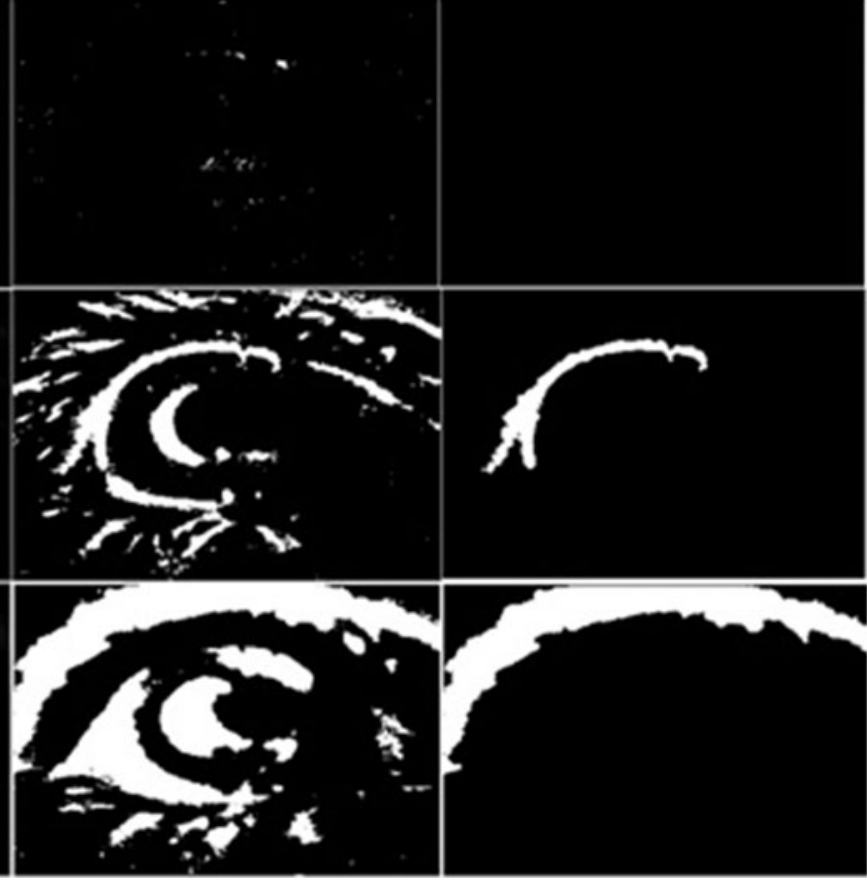

column; the third column displays the binary image transformed from the corresponding grayscale images; and the fourth column shows the biggest connected object found in the binary image

- Blink start The upper eyelid lowers to touch or cover part of the pupil (or the upper eyelid falls quickly but fails to touch the pupil, for some partially closed blinkers).

- Blink end The upper eyelid opens enough to release the whole pupil (or three video frames after start, for some partially closed blinkers).

According to the start and end criteria, the ten videos were watched to get the true blinks set.

The algorithm was run for different values of $\theta_{\mathrm{s}}, \theta_{\mathrm{e}}, \theta_{\mathrm{d}}$, and $\theta_{\mathrm{p}}$ to get different computer-generated blink sets. A comparison was then performed between the computergenerated blinks and the true blinks. Then true positives (TP), false positives (FP), true negatives (TN), and false negatives (FN) were obtained and receiver operating characteristic (ROC) curves were plotted to decide the best values of $\theta_{\mathrm{s}}, \theta_{\mathrm{e}}, \theta_{\mathrm{d}}$, and $\theta_{\mathrm{p}}$.

The thresholds $\theta_{\mathrm{s}}, \theta_{\mathrm{e}}, \theta_{\mathrm{d}}$, and $\theta_{\mathrm{p}}$ were determined sequentially. Initially they were empirically set to default values. The algorithm was run for different values of $\theta_{\mathrm{s}}-0.37,0.38,0.39$, 0.40 , and 0.41 - to get five computer-generated blink sets. Then the true blink set was matched to each computergenerated blink set to generate sets of variables for true and false positives and true and false negatives: TP, FP, TN, and $\mathrm{FN}$. Therefore, true-positive rates $\left[\mathrm{TPR}=\mathrm{TP} /(\mathrm{TP}+\mathrm{FN})^{*} 100\right]$ and false-positive rates $\left[\mathrm{FPR}=\mathrm{FP} /(\mathrm{FP}+\mathrm{TN})^{*} 100\right]$ were derived in order to plot the ROC curve; the best value of $\theta_{\mathrm{s}}$ was determined from the ROC curve. Similarly, from the obtained 
Table 1 True (TPR) and false (FPR) positive rates and accuracy, as percentages, for automatically identifying eye blinks with increasing values of $\theta_{\mathrm{s}}$, with $\left\{\theta_{\mathrm{e}}, \theta_{\mathrm{d}}, \theta_{\mathrm{p}}\right\}=\{3,5,78\}$

\begin{tabular}{lrrrrr}
\hline$\theta_{\mathrm{s}}=$ & 0.37 & 0.38 & 0.39 & 0.40 & 0.41 \\
\hline $\operatorname{TPR}(\%)$ & 92.1 & 91.1 & 90.5 & 90.3 & 82.4 \\
$\operatorname{FPR}(\%)$ & 0.3 & 0.3 & 0.2 & 0.1 & 0.1 \\
Acc(\%) & 99.5 & 99.6 & 99.6 & 99.7 & 99.6 \\
\hline
\end{tabular}

$\theta_{\mathrm{s}}$ and default values of $\theta_{\mathrm{d}}$ and $\theta_{\mathrm{p}}$, the best value of $\theta_{\mathrm{e}}$ was determined; $\theta_{\mathrm{d}}$ and $\theta_{\mathrm{p}}$ were then determined in the same way.

\section{Results}

In total, 270 true blinks were observed from the ten videos, with a mean blink frequency of $6.9 / \mathrm{min}( \pm 3.2 / \mathrm{min})$ and a mean duration of $179 \mathrm{~ms}( \pm 38 \mathrm{~ms})$. The results of the algorithm for the ten videos, with $\left\{\theta_{\mathrm{e}}, \theta_{\mathrm{d}}, \theta_{\mathrm{p}}\right\}$ set by default to $\{3,5,78\}$ and $\theta_{\mathrm{s}}$ set to $0.37,0.38,0.39,0.40$, and 0.41 , are reported in Table 1.

ROC curves were created using true-positive rates as the $y$-axis and false-positive rates as the $x$-axis, as is shown in Fig. 5 (the solid curve). According to the rule that "northwest is best" (Fogarty, Baker \& Hudson, 2005), $\theta_{\mathrm{s}}=0.40$ was the threshold that best balanced TPR and FPR.

Using $\theta_{\mathrm{s}}=0.40$ as the blink starting ratio threshold and $\left\{\theta_{\mathrm{d}}, \theta_{\mathrm{p}}\right\}$ defaults of $\{5,78\}$, we ran the algorithm with $\theta_{\mathrm{e}}=1$, $2,3,4$, and 5 , with the results shown in Table 2. The ROC curve was created as shown in Fig. 5 (the dashed curve). The ROC curve shows that the highest TPR was achieved when $\theta_{\mathrm{e}}=1$, and when $\theta_{\mathrm{e}}$ was increased, the TPR decreased,
Table 2 True (TPR) and false (FPR) positive rates and accuracy, as percentages, for automatically identifying eye blinks with increasing values of $\theta_{\mathrm{e}}$, with $\left\{\theta_{\mathrm{s}}, \theta_{\mathrm{d}}, \theta_{\mathrm{p}}\right\}=\{0.40,5,78\}$

\begin{tabular}{lrrrrr}
\hline$\theta_{\mathrm{e}}=$ & \multicolumn{1}{c}{1} & \multicolumn{1}{c}{3} & \multicolumn{1}{c}{4} & \multicolumn{1}{c}{5} \\
\hline $\mathrm{TPR}(\%)$ & 93.4 & 91.7 & 90.3 & 89.2 & 87.8 \\
$\mathrm{FPR}(\%)$ & 0.3 & 0.2 & 0.1 & 0.1 & 0.1 \\
Acc(\%) & 99.6 & 99.7 & 99.7 & 99.7 & 99.7 \\
\hline
\end{tabular}

but the FPR improved at the same time. Therefore, $\theta_{\mathrm{e}}=3$ was the threshold that best balanced the FPR and TPR.

Similarly, $\theta_{\mathrm{d}}$ and $\theta_{\mathrm{p}}$ were determined by plotting ROC curves, and we found $\theta_{\mathrm{d}}=5$ and $\theta_{\mathrm{p}}=78$.

With the determined values $\left\{\theta_{\mathrm{s}}, \theta_{\mathrm{e}}, \theta_{\mathrm{d}}, \theta_{\mathrm{p}}\right\}=\{3,0.40,5,78\}$, the overall performance of the algorithm was $\mathrm{TPR}=90.3 \%$, $\mathrm{FPR}=0.1 \%$, and accuracy $=99.7 \%$.

\section{Conclusions}

In this study, we developed an algorithm that captures eye blinks from videos obtained from head-mounted eyetrackers. We identified two major categories for blinks: completely closed eye blinks, in which the upper eyelid completely drops over the pupil during a blink, and partially closed eye blinks, in which the upper eyelid barely drops over the top of the pupil. Thus, any algorithm for detecting blinks accurately cannot rely on the pupil being covered during a blink. Our algorithm is simple but quite robust, showing high performance of $\mathrm{TPR}=90.3 \%, \mathrm{FPR}=0.1 \%$, and accuracy $=$ $99.7 \%$. The algorithm works by detecting the start and end of a blink by measuring the difference between consecutive
Fig. 5 ROC curves for sensitivity and specificity. The blue dashed curve was for determining $\theta_{\mathrm{s}}$, using $\left\{\theta_{\mathrm{e}}, \theta_{\mathrm{d}}, \theta_{\mathrm{p}}\right\}=\{3,5,78\}$; the red solid curve was for determining $\theta_{\mathrm{e}}$, using $\left\{\theta_{\mathrm{s}}, \theta_{\mathrm{d}}, \theta_{\mathrm{p}}\right\}=$ $\{0.40,5,78\}$

\section{ROC curves for determing starting and ending thresholds}

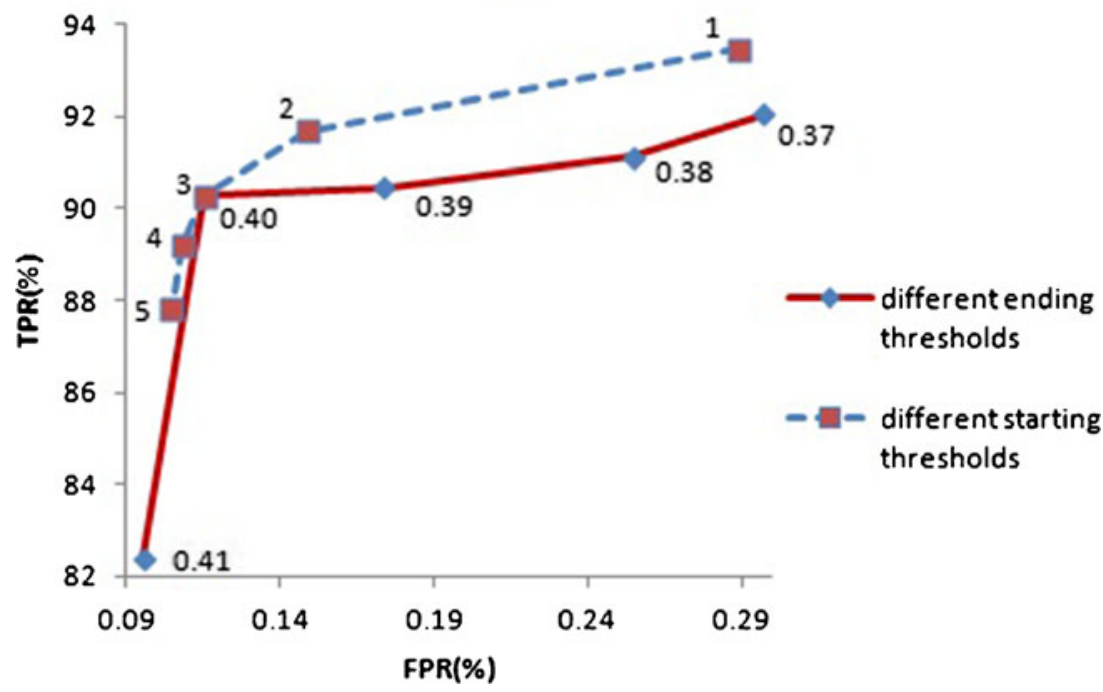


frames for the start point of a blink, and measuring pupil size for the end point.

Future work will extend this algorithm to work with regular webcams, which have less resolution and generate noisy data due to varying lighting conditions.

Author Note This research was supported by grants from the Canadian Natural Sciences and Engineering Research Council (NSERC) and the Royal College of Physicians and Surgeons in Canada (RCPSC).

\section{References}

Andreassi, J. L. (2000). Human behavior and physiological response (4th ed.). Mahwah, NJ: Erlbaum.

Benedetto, S., Pedrotti, M., Minin, L., Baccino, T., Re, A., \& Montanari, R. (2011). Driver workload and eye blink duration. Transportation Research Part F, 14, 199-208. doi:10.1016/j.trf.2010.12.001

Blumenthal, T. D., Cuthbert, B. N., Filion, D. L., Hackley, S., Lipp, O. V., \& Van Boxtel, A. (2005). Committee report: Guidelines for human startle eyeblink electromyographic studies. Psychophysiology, 42, 1-15. doi:10.1111/j.1469-8986.2005.00271.x

Bradski, G., \& Kaehler, A. (2008). Learning OpenCV: Computer vision with the OpenCV Library Retrieved from http://ebookee.org/LearningOpenCV-Computer-Vision-with-the-OpenCV-Library_192365.html

Caffier, P. P., Erdmann, U., \& Ullsperger, P. (2003). Experimental evaluation of eye-blink parameters as a drowsiness measure. European Journal of Applied Physiology, 89, 319-325.

Deuschl, G., \& Goddemeier, C. (1998). Spontaneous and reflex activity of facial muscles in dystonia, parkinson s disease, and in normal subjects. Journal of Neurology, Neurosurgery and Psychiatry, 64, 320-324.

Evinger, C., Manning, K. A., \& Sibony, P. A. (1991). Eyelid movements mechanisms and normal data. Investigative Ophthalmology and Visual Science, 32, 387-401.

Fogarty, J., Baker, R. S., \& Hudson, S. E. (2005). Case studies in the use of ROC curve analysis for sensor-based estimates in human computer interaction. Waterloo, British Columbia: Paper presented at Graphics Interface 2005.

Grauman, K., Betke, M., Gips, J., \& Bradski, G. R. (2001). Communication via eye blinks - detection and duration analysis in real time. Kauai, HI: Paper presented at the IEEE Computer Society Conference on Computer Vision and Pattern Recognition.

Haak, M., Bos, S., Panic, S., \& Rothkrantz, L. J. M. (2009). Detecting stress using eye blinks and brain activity from EEG signals. Prague, Czech Republic: Paper presented at the Driver Car Interaction \& Interface 2009.

Kong, X., \& Wilson, G. (1998). A new EOG-based eyeblink detection algorithm. Behavior Research Methods, 30, 713-719. doi:10.3758/ bf03209491
Lalonde, M., Byrns, D., Gagnon, L., Teasdale, N., \& Laurendeau, D. (2007). Real-time eye blink detection with GPU-based SIFT tracking. Regina, SK: Paper presented at the Fourth Canadian Conference on Computer and Robot Vision.

Marshall, S. P. (2007). Identifying cognitive state from eye metrics. Aviation, Space, and Environmental Medicine, 78 (5 Suppl), B165-B175.

Mitalis, S., \& Druss, F. (1985). An optoelectronic motion-sensing eye blink detector. Behavior Research Methods, 17, 385-387. doi:10.3758/bf03200946

Moriyama, T., Kanade, T., Cohn, J., Xiao, J., Ambadar, Z., Gao, J., \& Imanur, M. (2002). Automatic recognition of eye blinking in spontaneously occurring behavior. Quebec City: Paper presented at the 16th International Conference on Pattern Recognition (ICPR 2002).

Pedrotti, M., Lei, S., Dzaack, J., \& Rötting, M. (2011). A data-driven algorithm for offline pupil signal preprocessing and eyeblink detection in low-speed eye-tracking protocols. Behavior Research Methods, 43, 372-383. doi:10.3758/s13428-010-0055-7

Ryu, K., \& Myung, R. (2005). Evaluation of mental workload with a combined measure based on physiological indices during a dual task of tracking and mental arithmetic. International Journal of Industrial Ergonomics, 35, 991-1009. doi:10.1016/j.ergon.2005.04.005

Schiffman, H. R. (2000). Sensation and perception: An integrated approach (5th ed.). New York, NY: Wiley.

Smith, P., Shah, M., \& Lobo, N. D. V. (2000). Monitoring head/eye motion for driver alertness with one camera. Barcelona, Spain: Paper presented at the International Conference on Pattern Recognition (ICPR 2000).

Sukno, F. M., Pavani, S.-K., Butakoff, C., \& Frangi, A. F. (2009). Automatic assessment of eye blinking patterns through statistical shape models. In M. Fritz, B. Schiele, \& J. H. Piater (Eds.), Computer vision systems, Lecture Notes in Computer Science, vol. 5815 (pp. 33-42). Heidelberg: Springer. doi:10.1007/978-3-642-04667-4_4

Tien, G., Atkins, M. S., Zheng, B., \& Swindells, C. (2010). Measuring situation awareness of surgeons in laparoscopic training. In Proceedings of the 2010 Symposium on Eye-Tracking Research \& Applications (pp. 149-152). New York, NY: ACM

Veltman, J. A., \& Gaillard, A. W. K. (1996). Physiological indices of workload in a simulated flight task. Biological Psychology, 42, 323-342. doi:10.1016/0301-0511(95)05165-1

Veltman, J. A., \& Gaillard, A. W. K. (1998). Physiological workload reactions to increasing levels of task difficulty. Ergonomics, 41, 656-669. doi:10.1080/001401398186829

Wu, J., \& Trivedi, M. M. (2008). Simultaneous eye tracking and blink detection with interactive particle filters. EURASIP Journal on Advances in Signal Processing, 2008(823695), 1-17. doi:10.1155/2008/823695

Zheng, B., Jiang, X., Tien, G., Meneghetti, A., Panton, N., \& Atkins, M. S. (2012). Workload assessment of surgeons: Correlation between NASA TLX and blinks. Surgical Endoscopy, 26, 2746-2750.

Zheng, B., Tien, G., Atkins, S. M., Swindells, C., Tanin, H., Meneghetti, A., \& Panton, M. (2011). Surgeon's vigilance in the operating room. American Journal of Surgery, 201, 667-671. 